\title{
CALORIMETRY OF PHASE AND ORDERING TRANSITIONS
}

\author{
EDGAR F. WeSTRUM, JR. \\ Department of Chemistry, University of Michigan, \\ Ann Arbor, Michigan 48104, U.S.A.
}

\begin{abstract}
This terse review is concerned with experimental calorimetric progress in the study of a variety of transitions primarily by use of heat capacity and entropy as parameters of the energetic and of order-disorder phenomena in a variety of substances. Emphasis has been placed upon selecting interesting examples rather than providing encyclopaedic coverage.

Although calorimetric techniques generally improve gradually rather than discontinuously, several developments in recent years merit special attention. They are: (a) the improvement in the high-frequency relaxation (HFR) method for measuring the magnetic heat capacity; (b) a calorimeter for the measurement of small heat capacities below $1 \mathrm{~K}$ which permits measurement at a rate of 80 heat capacity points per hour; (c) a laser heat-pulse method for $30 \mathrm{mg}$ samples, and a dynamical temperature relaxation method from 2 to $20 \mathrm{~K}$; (d) simple high-pressure calorimeters for measurements in the cryogenic region; (e) the use of oxide melt solution calorimetry in the determination of enthalpies of transition at high temperatures. It is worth noting also that the laser calorimeter may provide a convenient means of ascertaining enthalpies of transition over a considerable range of temperature. Moreover, differential scanning calorimetry is rapidly coming of age and is enjoying an enhanced popularity. An analysis of the thermodynamics of organic substances studied during the last decade indicates that 60 of 168 compounds listed exhibit transitions in the solid state. Extensive studies on the thermodynamic properties of globular molecules continue to be reported; the systems involved often include plastically crystalline phases. The molecular freedom in crystals of polynuclear, condensedring, aromatic substances is an object of interest not only to calorimetrists but to solid-state chemists using other techniques as well. Azulene provides a different and thus far unique example of non-cooperative disordering behaviour. The glass transformations produced on undercooling organic liquids have been studied. Although a wealth of DSC data exist on these anisotropic liquid crystals and their transformations, sparse examples of equilibrium data have been reported. But in many respects, the ionic crystalline state is still the neglected one. Even the trend of the heat capacity in the vicinity of the melting transition has been studied for probably fewer than a dozen substances. Similarities
\end{abstract}

t The author expresses his appreciation for the cutrent financial support of his research endeavours to the National Science Foundation and to that previously provided by the U.S. Atomic Energy Commission over two decades. 
between the reorientational behaviour of alkali azides and alkali hydrogen difluorides have been pointed out with respect to both their phase behaviour and the thermophysics of the transformations involved. The Schottky anomaly (which has just passed its 50th anniversary) is becoming established as an increasingly significant component of the heat capacities of many lanthanide, actinide and transition element compounds. Finally, since time permits only limited coverage bibliographic volumes on phenomena related to phase transitions are summarized.

\section{INTRODUCTION}

Abrupt changes of the molecular freedom of a molecule in a crystalline solid are among the most fascinating and still least understood of physical problems. Such transitions are often accompanied by dramatic changes in physical characteristics of the substance disproportionate to the subtle structural metamorphoses which occur at the molecular level. Although many experimental techniques provide insight into the nature and the mechanism of molecular freedom in crystalline substances, calorimetric studies of heat capacity and related thermal phenomena have contributed much to the understanding of molecular motion and of transitions. Precise measurements of heat capacity and enthalpies of transition are now readily made by adiabatic calorimetry over the range of 0.3 to $600 \mathrm{~K}$ and with only somewhat increased difficulty to temperatures as high as $1500 \mathrm{~K}$.

The pre-eminence of the heat capacity as a revealing parameter of energetics at the molecular and crystalline level is enhanced, of course, as the temperature drops below the thermal chaos. At high temperatures the entropy (for example, of an order-disorder transition) becomes a significant and useful criterion and at the modern higher temperature region it may even become the dominant factor in the Gibbs energy equation since its contribution is multiplied by the absolute temperature.

To reduce the extent of the hundreds of publications bearing on the title topic to more manageable proportions, this review emphasizes the special interests of its author and by fairly arbitrary decisions minimizes the coverage of recent theoretical developments, correlations and calculations, and excludes any attempt at being truly encyclopaedic in scope. It stresses those aspects which represent especially significant or unique contributions or initiated new trends in the application of thermophysical techniques to the study of transitions of matter.

But even a cursory glance at the thermodynamics literature for the last few years will convince the reader of a remarkable change. Although the number of 'professional' thermodynamicists interested in the solid state is limited to a few hundred individuals the world over, the bulk of solid-state thermo. dynamic information now comes from the laboratories of a variety of solidstate scientists whose prime interest may be in some other field, but who, from time to time, generate thermodynamic data. 


\section{SIGNIFICANTLY IMPROVED CALORIMETRIC TECHNIQUES}

Although calorimetric techniques generally improve gradually rather than discontinuously, several developments in recent years are of sufficient significance io merit a special heralding.

High-frequency-relaxation method--The basis for the high-frequencyrelaxation (HFR) method for measuring the magnetic heat capacity, $C_{m}$, originated with Casimir and du Pré and uses purely magnetic measurements to determine $C_{\mathrm{m}}$ without the usual uncertainty, due to the generally much larger lattice heat capacity. It thus allows accurate estimates over wide ranges of temperature. The recent development of cryogenic tunnel diode oscillators by Skjeltorp and Wolf has been used to improve and extend this method ${ }^{1}$. At temperatures high compared with the onset of magnetic ordering, $C_{\mathrm{m}}$ can be fitted to a series expansion of the form $C_{\mathrm{m}} / R=C_{2} / T^{2}+C_{3} / T^{3}+\ldots$, in which $R=N k_{\mathrm{B}}$ and $N$ is the number of magnetic spins. This can be related to microscopic spin-spin interaction parameters, and additional information can also be obtained from the field dependence of $C_{\mathrm{m}}$ (which can be related to the temperature dependence of the isothermal susceptibility). Examples of materials for which this technique has been used successfully include rare earth halides, hydroxides and garnets. It has already been applied to the study of the magnetic and thermal properties of gadolinium and terbium hydroxides and gadolinium trichlorides ${ }^{2-4}$.

Rapid response cryogenic calorimetry A ${ }^{3} \mathrm{He}$ calorimeter with rapid response useful for the measurement of small heat capacities below $1 \mathrm{~K}$ has also been reported by Golbrecht et $a l^{5}$. This calorimeter employs a superconducting heat switches, achieves thermal equilibrium within $1-2$ seconds with metallic samples, and permits measurement of heat capacities as small as $30 \mu \mathrm{J} \mathrm{K}^{-1}$ with a precision of one per cent above $04 \mathrm{~K}$, at a rate of 80 heat capacity points per hour.

Cryogenic laser calorimetry-Heat capacities one or two orders of magnitude smaller than those typically measured in this range were involved during study on the antiferromagnetic ordering of praseodymium hexaboride in the range $2-20$ by Lee et al. ${ }^{6}$. They used the pulse technique of Morin and Maita $^{7}$ and found that the heat pulse method in the vicinity of the Néel point (near $7 \mathrm{~K}$ ), where the heat capacity was large, required correspondingly long times to establish thermal equilibrium after application of heat pulses. In order to ascertain the possible presence of an isothermal absorption of heat on a small $(30 \mathrm{mg})$ sample, a dynamical temperature relaxation method which employs heating by laser beams was developed by Lee et al. ${ }^{6}$ This involves the use of laser radiation to heat the sample, a technique which has been pioneered at higher temperatures ${ }^{8}$ in Japan.

In the experimental arrangement employed, a silicon bolometer with a thin diffusion-doped layer on one side was employed as temperature sensor. At low temperatures, the entire wafer except the diffused layer is insulating. The layer resistance was used as a means of indicating temperature of the sample (which was bonded to the insulating side by ultrasonic soldering). Electrical contact and mechanical support for the bolometer were established by means of fine gold wires thermally anchored to a copper block, the temperature of which can be varied with a heater and sensed with a thermocouple. 
Both sample and bolometer were heated by a laser beam directed down a light pipe into the evacuated cryostat immersed in liquid helium. The beam is switched on suddenly and the time constant of the resulting thermal relaxation is used to deduce the heat capacity. By operating a bolometer in a constant current mode, they were able to describe the small exponentially saturating temperature produced by the incident radiation in terms of a time constant. A determination of the time constant then provides an absolute measurement of heat capacity, provided thermal equilibrium between bolometer and sample is established in a time short compared with the time constant.

To verify that this was indeed the case, a variant on the AC-temperature technique ${ }^{9}$, in which the laser radiation is chopped at fixed frequencies and the AC-component of the bolometer voltage is detected with a lock-in amplifier, was used. The frequency dependence of the $\mathrm{AC}$-signal then provides a measure of the internal time constant.

Measurements at decreasing temperature-Ehrat and Rinderer $^{10}$ have devised a calorimetric device in which silicone grease provides thermal contact between metallic samples and refrigerant and permits determination of both the heat capacity and the coefficient of thermal conductivity of the grease as the temperature is lowered over the range $1.5-4.2 \mathrm{~K}$. This approach would appear to be of particular value in the study of superconductive transitions in a magnetic field.

Quenching of phases stable at higher temperature-It is often desirable to obtain a metastable phase by quenching a phase stable at higher temperatures. Glasses or vitreous phases may be obtained from many organic or molecularly crystalline substances by cooling at the rate of $20 \mathrm{~K}$ per minute ${ }^{11}$ and it is occasionally desirable to undercool a phase stable at higher temperatures in order to ascertain transitional increments of thermodynamic functions at the reference temperature $0 \mathrm{~K}$. For example, such information on undercooled plastically crystalline phase I adamantane would be desirable to resolve the discrepancy between the crystallographic interpretation of the mechanism of the plastic-crystal transition and an entropic one based upon low-temperature calorimetric data. Heat capacities on the undercooled phase from near $0 \mathrm{~K}$ would permit the evaluation of the entropy of transition at absolute zero and thereby free it from unknown contributions from vibrational frequency changes at the transition temperature ${ }^{12}$.

To achieve quenching of samples, an adiabatic, anetoid cryostat of the type used between 5 and $350 \mathrm{~K}$ has been modified to permit breaking the vacuum in the space between the lowest-temperature refrigerant tank and the calorimeter without interference with the insulating vacuum in the other parts of the cryostat. This is achieved by means of an indium-tin gasketed seal and potting the electrical leads in epoxy as they leave this region. In addition, provision is made so that a stream of (purified) liquid nitrogen or of liquid helium may be vented through the adiabatic shield enclosure directly onto the calorimeter in order to provide extremely rapid cooling of the sample ${ }^{12}$. These features have also been provided in the cryostat for the miniaturized calorimeter described earlier.

High-pressure calorimetry-One of the least popular variables among calorimetrists of all temperature persuasions seems to be pressure. However, 
a simple high-pressure calorimeter has been devised by Smith and Phillips ${ }^{13}$. This calorimeter consists of a small piston and cylinder arrangement with a mass of about $150 \mathrm{~g}$, constructed entirely from high-purity copper-beryllium alloy ( 2.1 weight per cent beryllium) which was heat treated to full hardness. The sample was placed in a close-fitting Teflon sleeve between two Teflon discs. The cell is supported by an external screw-on collar and pressure is applied to the sample at room temperature in a standard press. At the required pressure, the locking screw is tightened down onto the internal piston and the calorimeter is removed from the press and is installed in a ${ }^{3} \mathrm{He}$ cryostat. Since the heat capacity of the sample comprises typically only three to five per cent of the total measured heat capacity, reasonably high precision is needed in the measurement of heat capacities. This device has been used for studies of the superconductivity of $\alpha$-uranium to pressures as high as $10 \mathrm{kbar}$.

Computerization-Science, like haute couture, has its fashions. Automation these days enables one to load one's calorimeter, press the button, go to lunch or to Monte Carlo (depending on the duration of the experiment and one's financial status) and when one does come back to the desk one may pick up the computer output with all the relevant data transposed into a format suitable for printing the table of data and possibly of thermodynamic functions. In most instances, some degree of semiautomatic and semimanual operation seems to be the preferred solution but in some instances an on-line computer may actually be used to operate the calorimeter.

\section{MOLECULAR CRYSTALS-EXOTIC PHASES}

Molecular phases-An analysis by Martin ${ }^{14}$ of the thermodynamics of organic substances made during the last decade indicates that 60 of 168 compounds listed exhibit transitions in the solid state. Extensive studies on the thermodynamic properties of globular molecules continue to be reported ; the systems involved often include plastically crystalline phases. Interestingly interpreted order-disorder transitions in these substances occur and are consistent in general with extant theories. Additional penetrating analyses by Brot and his co-workers ${ }^{15,16}$ have extended the theoretical aspects of partial disorder in crystals, reorientational movements of molecules, and phase transitions in to better accord with experiment.

Ortho- and meta-carboranes (plastic crystals par excellent) have been studied and reasonable interpretation of their transitional entropy increments made ${ }^{17}$.

Critical points-Measurements of the heat capacity at saturated vapour pressure of the system methane-argon near its solid-solid consolute point ${ }^{18}$ have revealed striking anomalies and evidence of probable suppression of the anomaly by lattice strain. Special difficulties associated with accurate measurements of the heat capacity in the vicinity of its critical point have been enumerated and include effects due both to gravity and to critical slowing down ${ }^{19}$.

Liquid crystals-Although melting to a smectic or a nematic liquid crystal phase is one of several ways some crystals may depart from their phases. all too little equilibrium calorimetry has been performed to ascertain the energetics, order and thermodynamic behaviour through such transformations. 


\section{EDGAR F. WESTRUM, JR.}

Some comment on the enhanced role of differential thermal analysis and differential scanning calorimetry is needed. These techniques of tremendous significance in survey measurements are, however, occasionally interpreted as being more definitive than circumstances warrant. Equilibrium measurements will prove important for some phenomena.

The heat capacity $(300-500 \mathrm{~K})$ and transition thermodynamics of an extended range high-temperature nematic liquid crystal have been measured ${ }^{20}$ by equilibrium adiabatic calorimetry (cf. Figure 1). These authors report that

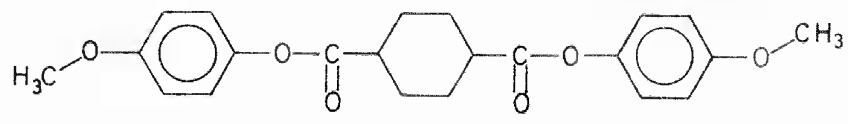

DMC

Figure 1. The structure of di-(p-methoxy)-trans-cyclohexane-1,4-dicarboxylate ${ }^{20}$.

di-(p-methoxyphenyl)-trans-cyclohexane-1,4-dicarboxylate melts at 416.077 $\left[\Delta S_{f} / \mathrm{J} \mathrm{mol}^{-1} \mathrm{~K}^{-1}=75.6\right]$ to a nematic phase which clears at $516.0 \mathrm{~K}$ $\left[\Delta S_{t} / \mathrm{J} \mathrm{mol}^{-1} \mathrm{~K}^{-1}=5.6\right]$. A solid state phase transition is apparent at $411.48 \mathrm{~K}\left[\Delta S_{t} / \mathrm{J} \mathrm{mol}^{-1} \mathrm{~K}^{-1}=10.6\right]$ and the authors interpret this as an $R$ In $4\left[\Delta S_{t}=11.5 \mathrm{~J} \mathrm{~mol}^{-1} \mathrm{~K}^{-1}\right]$ order-disorder transition due to restricted rotation of the terminal methoxy groups about the ring-to-oxygen bond. These results are of especial interest for two reasons. First, the material proved sufficiently stable to allow purification by zone-refining. This research represents the first thermodynamic investigation of a well-characterized liquid crystal sample (purity $99.84 \%$ ). Second, the results obtained in the nematic range allow useful comparisons to be made with various theoretical predictions.

The glassy phase-Interest in the vitreous state and its relevance to inclusion with crystal thermodynamics centers primarily around the lowtemperature heat-capacity difference between glasses and crystal and its interpretation, as well as, of course, the glass-crystal transformation and residual entropy. Recent heat capacities at low temperatures have been measured on vitreous glycerol (used as a thermal contact medium), vitreous dimethylbutane, cyclohexanol, crystalline and 'glassy-crystalline' hexanol, arsenic trifluoride, germanium, and beryllium diflouride as well as on high polymers. Professor Seki's group at Osaka have been especially productive in the study of vitreous phases.

\section{POLYNUCLEAR-CONDENSED-RING AROMATICS}

Azulene and complex compounds-Early crystallographic studies ${ }^{21-26}$ led to disagreement among investigators as to whether or not the azulene crystal was disordered at room temperature. The controversy seems to have been satisfactorily resolved ${ }^{27,28}$ in favour of space group $P 2 / / a$. The disorder arises from a random distribution of azulene molecules among two possible orientations at each lattice site, one differing from another by a $180^{\circ}$ rotation 
about an axis perpendicular to the plane of the molecule. Günthard originally proposed this model to account for his $\mathrm{x}$-ray diffraction results ${ }^{29}$, later citing supporting evidence for disorder based upon vapour pressure measurements and gas phase entropy values calculated from infrared spectral data ${ }^{30}$. He suggested the probable existence of an $R$ in 2 contribution to the azulene crystal entropy accompanying a transition from a low-temperature ordered phase to a higher-temperature disordered phase, a transition that should manifest itself in the heat capacity-temperature curve.

The molal heat capacity of azulene over the temperature range $5-400 \mathrm{~K}$ has been measured by adiabatic calorimetry. No evidence for a cooperative transition (other than melting) was found. A check residual entropy at $0 \mathrm{~K}$. using vapour phase entropies of Kovats et al. ${ }^{30}$ and vapour pressure data of Bauder and Günthard ${ }^{31}$, indicated $\Delta S^{\circ}=-0.4 \mathrm{cal} \mathrm{mol}^{-1} \mathrm{~K}^{-1}$ at $0 \mathrm{~K}$ to within $\pm 0.8 \mathrm{cal} \mathrm{mol}^{-1} \mathrm{~K}^{-1}$ (the limits of uncertainty in the vapour pressure and gas entropy data). Thus any disorder existing at room temperature would appear to be absent at very low temperatures, but the uncertainty is a matter of concern.

Comparison of values for the crystal entropy of azulene with those of its isomer, naphthalene, reported by McCullough et al. ${ }^{32}$ over the temperature range $0-353.43 \mathrm{~K}$ (the triple point of naphthalene) reveals a steadily increasing excess entropy for azulene over naphthalene with rising temperature. The excess reaches a maximum value of 1.60 near the triple point of naphthalene. Except for the high activation energy, it would be tempting to interpret this excess entropy as arising from a gradual onset of orientational disorder in azulene beginning at very low temperatures and reaching a value of $R \ln 2$ (1.38) near the triple point of naphthalene. The implicit assumption that the lattice contribution to the total entropy is essentially the same for azulene and naphthalene is not surprising in view of their close similarity in crystal structure.

Recent nuclear magnetic resonance studies on solid azulene by Fyfe and Kupferschmidt ${ }^{33}$ indicate that azulene molecules are in dynamic equilibrium between two alternative orientations. The motion is a $180^{\circ}$ libration about an axis perpendicular to the plane of the molecule. However, they report evidence for a transition centered at $310 \mathrm{~K}$ and predict that a structure determination below $270 \mathrm{~K}$ should show a single molecular orientation rather than disorder. No evidence for a transition in this region was found by van Tets and Günthard ${ }^{34}$, who measured the temperature dependence of the infrared spectrum of solid azulene between 113 and $371 \mathrm{~K}$, a finding that is consistent with the absence of evidence for a cooperative transition in the heat capacity dependence on temperature described herein.

Reported order-disorder transitions extending over temperature ranges as broad as this one apparently does and lacking a peak in the heat capacitytemperature curve are unknown. Similar behaviour may be expected in other substances which exhibit disordered crystal structures at higher temperatures, i.e. $p$-chlorobromobenzene and the azulene-trinitrobenzene complex. The azulene-trinitrobenzene complex is an interesting comparison molecule ${ }^{35}$ differing in the presence of an energy increment between its two orientations and hence may be anticipated to have a temperature dependent ordering [cf, Figure 2]. 
EDGAR F. WESTRUM, JR.

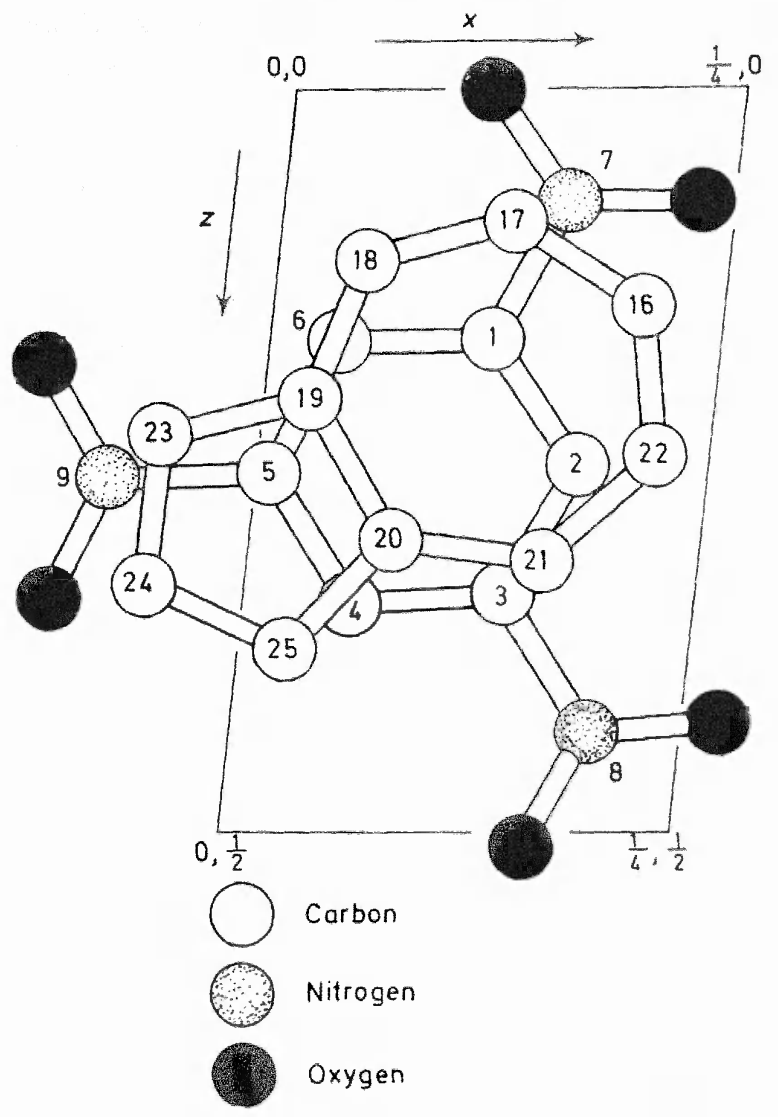

Figure 2. Projection of the molecular structure of the azulene-s-trinitrobenzene complex ${ }^{35}$.

\section{ELECTRONIC AND/OR MAGNETIC TRANSITIONS}

The Schottky anomaly-This artifact (which has just passed its 50th anniversary of recognition) is becoming established as an increasingly significant component of the heat capacities of many lanthanide, actinide and transition element compounds. Its morphology, its relationship to Einstein and Schottky functions, and its algebraic ramifications have been explored by Lyon and Westrum ${ }^{36}$ and its presence revealed in the behaviour of actinide sulphides of Grønvold et al. ${ }^{37}$. The resolution of the misfits between calculated Schottky anomalies and experimentally observed ones has been explained in terms of the expansion of the lattice and the spin lattice coupling ${ }^{38}$.

Enhanced appreciation of the significance of Schottky anomalies in transition metals and impurity modes is revealed by an especially interesting Schottky anomaly which arises near $0.2 \mathrm{~K}$ as a consequence of the $10^{-9} \%$ hydroxyl ion in sodium fluoride ${ }^{39}$. The sixfold orientational degeneracy is split due to tunnelling into a singlet, triplet and doublet ${ }^{40}$. The data show how certain impurities, which at concentrations as low as 0.1 p.p.m. would be very 
difficult to detect by other means, (e.g., optical spectroscopy), can be observed and even quantitatively determined by heat capacity measurement. They also reflect the great care required for the preparation of samples for heat capacity determinations.

A nuclear Schottky anomaly in antiferromagnetic vanadium sesquioxide ${ }^{41}$ has been reported below $0.5 \mathrm{~K}$. It is seldom realized that Schottky anomalies occur not only in the heat capacity but in the thermal expansion and other properties as well. More exotic results include Van Hove singularities in the heat capacities of crystals ${ }^{42}$.

Schottky anomalies in lanthanide sesquioxides--Although resolution of the levels which result from splitting the ionic ground state by the crystalline fields is usually achieved by spectroscopists, on occasion the cryogenic calorimeter can do yeoman service, especially in the absence of optical grade samples. By analysis of the Schottky anomaly in neodymium sesquioxide, for example (using the isostructural lanthanum oxide to evaluate the lattice heat capacity), the lowest levels were deduced ${ }^{43}$ as $0,21,81$ and $400 \mathrm{~cm}^{-1}$, in sharp constrast with previous theoretical predictions. Subsequently, the spectrum of neodymium(III) ion in a lanthanum(III) ion in an oxide host was observed $^{44}$ as $0,23,84,253$ and $496 \mathrm{~cm}^{-1}$. The first two excited levels are in excellent accord with corresponding values deduced from heat capacity data alone $\left(0,21,81\right.$ and $\left.400 \mathrm{~cm}^{-1}\right)$, and the centre of density of the higher levels also corresponds well. The interpretation of higher cubic lanthanide sesquioxides involves two non-equivalent types of cation sites. Three-fourths of the cations have $C_{2}$ symmetry. The remaining cations possess $C_{3 i}$ symmetry. Contributions to the electronic heat capacity, $C_{\mathrm{e}}$, based on spectroscopic levels reported for the $C_{2}$-type ions in erbium sesquioxide ${ }^{45}$, are represented by dashed curves in Figure 3, which depicts $C_{\mathrm{el}}$ for the low-temperature region. These values were derived by subtracting the lattice contribution from the apparent heat capacities. The balance of $C_{\mathrm{el}}$ remaining after removal of the contributions of the $C_{2}$ ions is attributed to the levels in the $C_{3 i}$ ions. The levels and their degeneracies are deduced in part from the position and magnitude of the Schottky peaks in the observed $C_{\mathrm{el}}$ (for separations of less than $100 \mathrm{~cm}^{-1}$ ) and in part from the splittings of the ground state term by fourth- and sixthorder terms of cubic crystal fields. The levels thus derived are doublets at 0 , $12,50,55,150$ and $450 \mathrm{~cm}^{-1}$ and a quartet at $550 \mathrm{~cm}^{-1}$. These correspond most closely to the levels in a cubic field with a ratio of sixth- to fourth-order terms $\left(V_{6} / V_{4}\right)$ of -0.25 . The $\Gamma_{8}$ level is split to 12 and 50 (or 55 ) $\mathrm{cm}^{-1}$, and the second $\Gamma_{8}$ level is split to 150 and $450 \mathrm{~cm}^{-1}$. The $C_{\mathrm{el}}$ data require levels at 12 , 50 and $55 \mathrm{~cm}^{-1}( \pm 10 \%$ of $\Delta E)$. Note that the resolution of high temperature drop calorimetric values (cf. ref. 46) and the experimental excess heat capacity to $1100 \mathrm{~K}$ is in good accord with theory.

Para- and ferroelectric transitions-The thermodynamics of lowtemperature electrocaloric phenomena in alkali halides doped with polar impurities has been formulated ${ }^{47}$ and found to accord with heat capacity data on rubidium chloride doped with cyanide ion, adiabatic depolarization temperature changes and the electrocaloric coefficient for potassium chloride doped with hydroxyl ion ${ }^{48}$. Recent progress in the phase transitions of orderdisorder ferroelectrics ${ }^{49}$ and a general treatment of the thermodynamics of broadened ferroelectric phase transitions ${ }^{50}$ have been reported. 
EDGAR F. WESTRUM, JR.

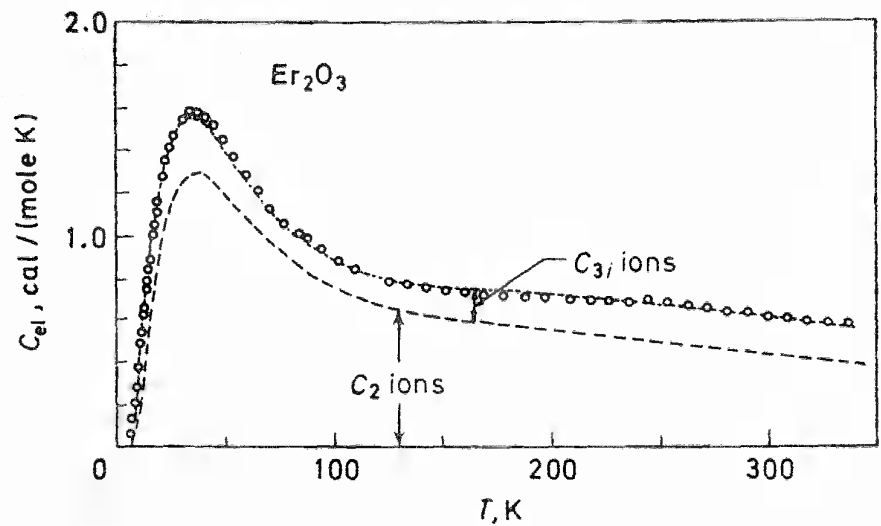

(a)

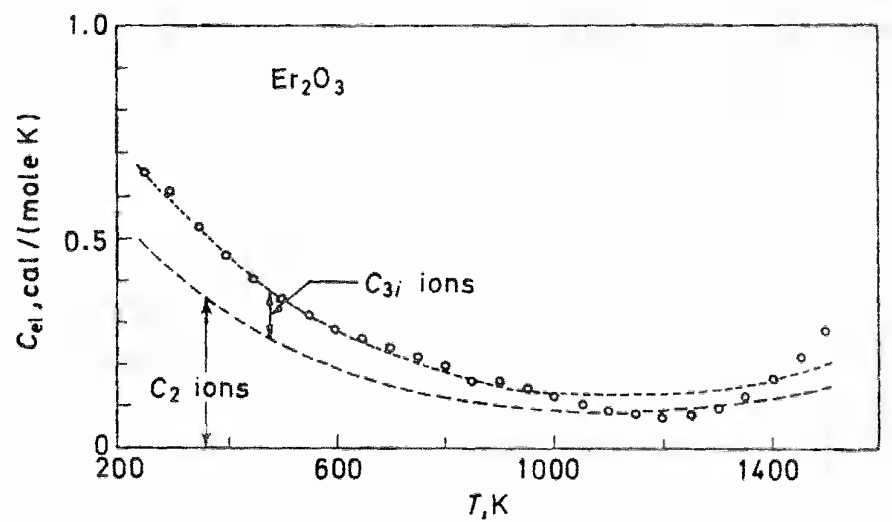

(b)

Figure 3. Schottky, (excess) heat capacity of $\mathrm{Er}_{2} \mathrm{O}_{3}{ }^{43}$.

Semi-, super- and metallic conductivity transitions-Thermodynamics of type II superconductors including the results of phenomenological theories and comparison with experimental values have been compiled ${ }^{51}$ and predictions of the effect of impurities on the singularities in the heat capacity of superconductors ${ }^{52}$ made by utilization of Ginzberg-Landau parameters. The important semiconductor $\rightleftarrows$ metal 'phase' transition has been explored by Hyland ${ }^{53}$.

Electronic heat capacity trends in the transition metals have been correlated with $d$ electron configurations ${ }^{54}$. Moreover, recent developments on the Verwey transition in magnetite ${ }^{55-57}$ and the Morin transition in hematite ${ }^{58}$ have resulted in unanticipatedly complex trends.

Magnetic (?) transitions-An interesting trend in the temperature dependence of the heat capacity near $0 \mathrm{~K}$ has become evident in recent studies on tetrauranium enneaoxide $\left(\mathrm{UO}_{2.250}\right)^{59}$. It is evident from Figure 4 that up to $10 \mathrm{~K}$ the heat capacity is much higher than that of uranium or thorium dioxide. The lattice contribution of both uranium enneaoxide and dioxide in this 


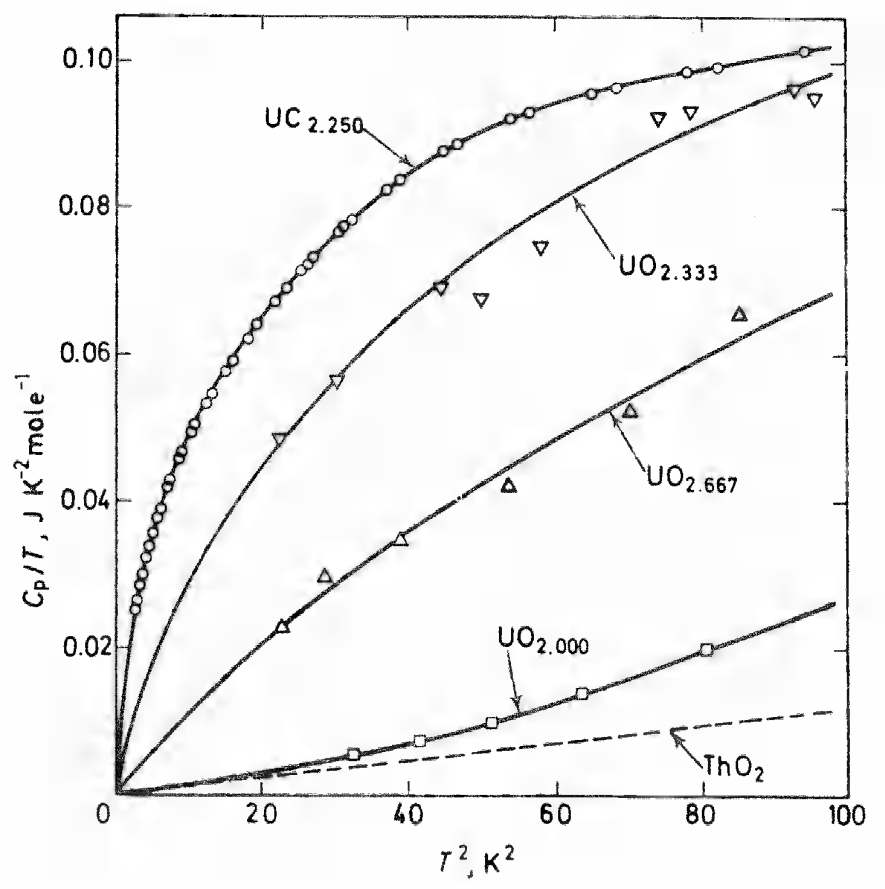

Figure 4. $C p / T$ vs. $T^{2}$ for several actinide oxides 59

temperature region can be well approximated by the heat capacity of thorium dioxide since these compounds have similar structures and nearly the same lattice constants and cationic masses. The spin-wave contribution in uranium dioxide becomes very large as the temperature approaches $30.4 \mathrm{~K}$, the Néel temperature. In the enneaoxide there is an additional contribution from the extra 0.250 moles of oxygen atoms, but this is probably negligible below $20 \mathrm{~K}$. A nother estimate of the lattice heat capacity of this substance may be obtained from values of the lattice constants at $300 \mathrm{~K}$ from which $\Theta_{\mathrm{D}}$ is calculated as $200 \mathrm{~K}$. Hence, the lattice heat capacity of the enneaoxide is 1.5 times the heat capacity of thorium dioxide, up to $10 \mathrm{~K}$. Regardless of which estimate of the lattice heat capacity of the enneaoxide is taken, it is evident that there is a large capacity contribution from other sources presumably magnetic. After deduction of the lattice contribution, the remaining heat capacity, $C_{m}$, for the enneaoxide is well represented by the relationship: $C_{\mathrm{m}}=(0.0155) \mathrm{T}^{2} \mathrm{~J} \mathrm{~K}^{-1}$ $\mathrm{mol}^{-1}$. No theoretical explanation of this interesting temperature dependence is known. Similar behaviour obtains in the uranium $\alpha-\beta$ - and $\gamma$-trioxides ${ }^{60}$.

Magnetic ordering ${ }^{61}$, first-order phase transitions ${ }^{62}$, the Curie point heat capacity of gadolinium metal ${ }^{63}$ and the Neel point heat capacity of $\mathrm{CoO}^{64}$ are typical of recent endeavours in this area. Measurement of the trend in the heat capacity of nickel in the region of its Curie point in increments of less than $0.01 \mathrm{~K}$ has been achieved by combining heating by chopped infrared light, sensing the temperature variations by Peltier effect, and phase-locked amplification ${ }^{65}$. 
Tungstates and molybdates-Analysis of the excess entropy associated with the para- to anti-ferromagnetic ordering transition in ferrous tungstate at $75.25 \mathrm{~K}$ yields an entropy of transition of $3.20 \mathrm{cal} \mathrm{mol}^{-1} \mathrm{~K}^{-1}$ (near $R \ln 5$ ) ${ }^{66}$. This value is in agreement with a 'spin-only' picture of ionic energy levels. Well above this Néel temperature, $T_{N}$ additional excess heat capacity occurs in comparison with that of isostructural zinc tungstate, consistent with a Schottky contribution arising from orbital splitting of the ${ }^{5} T_{2 g}$ term $\left(O_{h}\right.$ field) by low-symmetry ligand field components. These levels were calculated to lie $1500-2700 \mathrm{~K}$ above the ground term. This picture is remarkably similar in both quantitative and qualitative aspects to that for ferrous fluoride found earlier by Stout and co-workers. (For the fluoride: $T_{\mathrm{N}}=78.35 \mathrm{~K}$, $S_{\mathrm{m}}=R \ln 5$.) Further work on other transition metal tungstates (e.g. manganese, cobalt and nickel tungstate) is planned for the near future. In ferrous molybdate(II), a rounded lambda anomaly was found at $43.0 \pm 0.1 \mathrm{~K}$ (Néel point). The excess entropy associated with the anomaly appears close to $R \ln 2$. The rounding is probably a result of impurities produced by slow air oxidation. Studies on two independently produced samples of $\alpha$-ferrous molybdate also revealed rounded transitions at $31.6 \pm 0.1 \mathrm{~K}$.

High-resolution heat capacity measurements on single crystal cobalt dichloride hexahydrate ${ }^{67}$ below $4 \mathrm{~K}$ near magnetic phase transitions permitted determination of the critical exponents ${ }^{68}$. Low-spin-high-spin transitions have been modelled ${ }^{69}$. The implications of soft modes for phase transition behavior are being treated more intensively too ${ }^{70}$.

\section{TRANSITIONS-MAINLY IN IONIC CRYSTALS}

Transitions and energetics of phase behaviour-Brewer's proposed methods for predicting the crystal structures, mutual solubilities and multicomponent phase diagrams for the thirty elements of the first three transitions series ${ }^{71}$ and extensions ${ }^{72-74}$ involve correlation of electronic and crystal structures, and spectroscopically derived energies of various electronic states of gaseous atoms, as well as certain aspects of regular solution theory. His treatment provides elegant examples of the interrelation of energetics and bonding as well as considerable thermodynamic insight into the importance of various factors involved in metallic systems.

The energetics of crystals are related to those of other phases through phase transitions, e.g. sublimation, melting, solid-solid, crystal-glass, crystal-liquid crystal, etc. However, the tremendous growth in accurate knowledge about the structure and properties of crystals has not been paralleled by developments on the mechanism of melting. In fact, Ubbelohde's Melting and Crystal Structure ${ }^{75}$, in which liquids are described in terms of 'melts' of quasicrystalline models and anti-crystalline models related to them, is still in many respects a reasonable delineator of the state of the art. Drop-calorimetric enthalpies of melting for III/V, IV/V and V/VI compounds have been reported ${ }^{76}$; a dearth of adiabatic calorimetric data on the details of the melting transition still persists. Comparison between experiment and theory in Phase Transformations ${ }^{77}$ in metals and alloys in which extensive agreement has been attained does not, however, imply that the essential features of the field can be considered to be understood. Related progress on many types of 


\section{CALORIMETRY OF PHASE AND ORDERING TRANSITIONS}

crystalline materials has been detailed in other symposium proceedings ${ }^{78-83}$.

Ionic crystals - In many respects the ionic crystalline state is still the neglected one! The trend of the heat capacity in the vicinity of the melting transition has been studied for probably fewer than a dozen substances and solid-state transitions have also been little studied. Similarities between the reorientational behaviour of alkali azides and alkali hydrogen difluorides have been pointed out with respect to both their phase behaviour and the thermophysics of the transformations involved ${ }^{84}$. Reorientations of both linear anions have been found to occur at random either along the body diagonal or (for some of the heavier alkali compounds) along the crystallographic axes. In caesium hydrogen difluoride, a transition only approximately $3 \mathrm{~K}$ below melting is as yet unexplained ${ }^{85}$. Ammonium hydrogen difluoride does not show the solid-state transitional phenomenon but its enthalpy of melting has been determined ${ }^{86}$ to be about 25 per cent higher than that previously reported by Sudarik ov et al. ${ }^{87}$ by cryoscopy. An unusually sharp but definable first-order transition has been found in ammonium bromide at $412.67 \mathrm{~K}^{88}$ [cf. Figure 5].

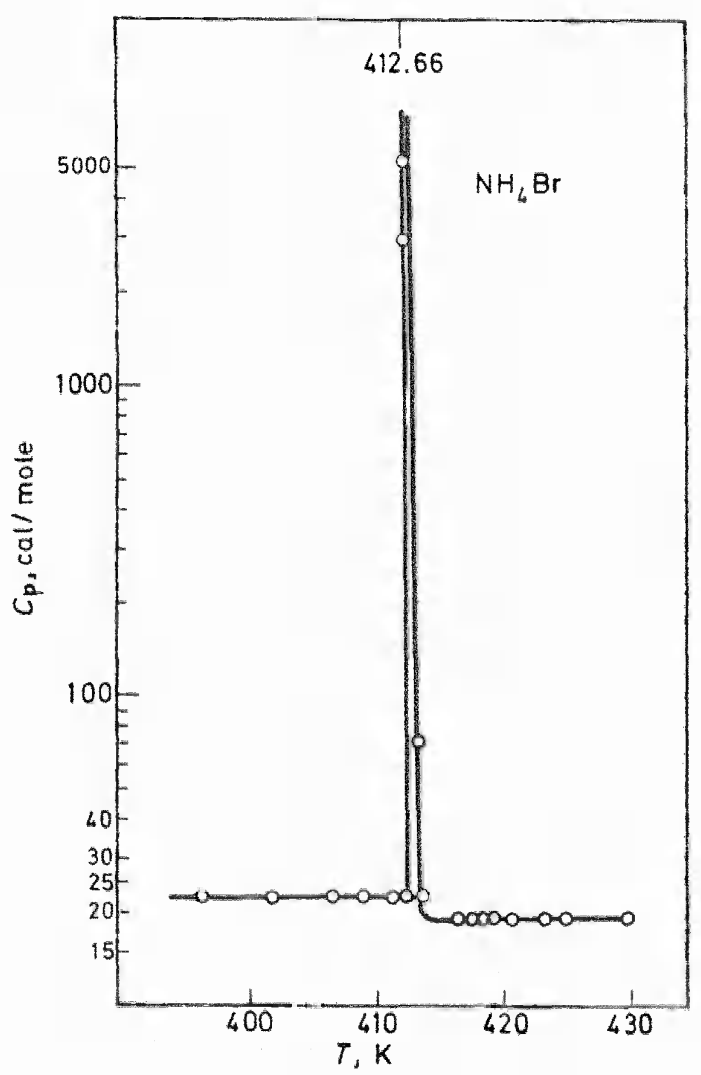

Figure 5. High-temperature transition in $\mathrm{NH}_{4} \mathrm{Br}^{88}$. 
Pressure constraints-High' pressure is even at present an unpopular and largely neglected constraint in the measurement of both heat capacity and transition thermophysics. Certainly formidable problems exist, especially for transitions where significant volume increments occur. Some initial endeavours are, however, worthy of note. For example, the heat capacity anomaly in ammonium chloride near $243 \mathrm{~K}$, discovered in 1922 by Simon, has been a prototype for the study of order-disorder transitions in crystals for many years. Although numerous experimental and theoretical studies have been devoted to the transition and the basic mechanism is well understood, none of the attempts to classify and totally explain the characteristic features are completely successful. In an exciting pioneering investigation by Trappeniers $^{89}$, the influence of pressure on the $\lambda$-point was studied by means of the differential thermoanalysis technique under conditions of hydrostatic pressures up to $3 \mathrm{kbar}$. He concluded that the $\lambda$-transition collapses under pressure and as a result the enthalpy change and the associated entropy increment are strongly reduced (i.e. they drop to less than 25 per cent of their value at one atmosphere within the $2 \mathrm{kbar}$ range).

More recent studies have been made on this transition, which occurs at about $242 \mathrm{~K}$ and is occasioned by the disordering of the ammonium tetrahedra in the chloride ion lattice, by Paukov et al. ${ }^{90}$. These investigators examined the heat capacity in the $200-320 \mathrm{~K}$ range under both ambient and elevated pressures as high as $3.6 \mathrm{kbar}$ and used the pressure as a parameter to vary the coupling of the lattice to the system being disordered. Their more precise measurements enabled them to considerably reduce the error in the measurements at high pressures. Their sample was compressed in silicon oil and pressure measured with a manganin wire. The pressure was static and adjusted prior to the beginning of the measurements. Apparently, their measurements were not made under totally equilibrium conditions. They concluded that, at pressures about $3 \mathrm{kbar}$, the heat capacity increased only by a factor of two whereas under normal pressure it rose by a factor of $30-40$. Thermograms indicated that the isothermal part of the enthalpy of transition was between 10 and $13 \mathrm{cal} \mathrm{mol}^{-1}$ except at the highest pressure. Unfortunately, similar studies are not available for a variety of other transitions in molecular and ionic crystals, nor are the measurements reported to date truly equilibrium values. They are suggestive, however, and are indicative of the need for further measurements of thermophysical properties at elevated pressures.

Other related literature-Brout's ${ }^{91}$ statistical theoretical presentation of transitions to experimental physicists has been supplemented in the recent past by Stanley's exposition ${ }^{92}$ (though largely limited to a description of magnetic and liquid-gas phase transformations) and the two-volume encyclopaedic treatment by Domb and Green ${ }^{93,94}$ of transitions and critical phenomena. The first volume ${ }^{93}$ pertains to exact solutions of ferromagnetic and ferroelectric models in one and two dimensions, emphasizing rigorous results and general theorems. The second volume ${ }^{94}$ provides a coherent survey on scaling, surface and finite size effects, and the dynamics of Ising models.

Finally, since time permits only limited coverage, it should be noted that the bibliographic volumes entitled Cooperative Phenomena Near Phase Transitions, $A$ Bibliography With Selected Readings ${ }^{95}$ provides a coded bibliography of some 2500 articles plus fifty relatively recent conference proceedings dealing 
with aspects of phenomena related to structural phase transitions as well as to critical points in liquid helium, in liquid crystals and in biological systems, as well as in cooperative phenomena in binary systems. The bibliography is current to June 1972. In addition, 35 experimental articles have been reproduced in full. Should the sceptic regard the study of phase transitions as passé or complete, then a quick perusal will disillusion him.

Jayaraman's timely review ${ }^{96}$ of the influence of pressure on phase transitions is pertinent and critical.

\section{CONCLUDING REMARKS}

Unquestionably, many important contributions have been slighted in this lecture and, indeed, emphasis has been placed on the areas of greatest familiarity to the author. But as Dr Samuel Johnson (on completion of his Dictionary in 1755) quipped, '. . In this work, when it shall be found that much is omitted, let it not be forgotten that much likewise is performed.' The extent to which we have enhanced our appreciation and understanding of the thermophysics and mechanisms of transitional behaviour since the Second International Conference on Thermodynamics and Thermochemistry has been delineated. In many instances, lacunae have been pointed out but one cannot escape the feeling that we are still looking at a fertile field of potential investigation spiced with many intriguing prospects for future endeavour.

\section{REFERENCES}

1 A. T. Skjeltorp and W. P. Wolf, Phys. Rev. to be published.

A. T. Skjeltorp, C. A. Catanese, H. E. Meissner and W. P. Wolf, Phys. Rev to be published.

C. A. Catanese, A. T. Skjeltorp, H. E. Meissner and W. P. Wolf, Phys. Rev to be published.

R. B. Clover and W. P. Wolf. Solid State Commun. 6, 331 (1968).

K. H. Golbrecht. J. J. Veyssie and L. Weil. Anm. Acad. Sci. Feinn, Ser. A VI. 210, 63 (1966).

K. N. Lee, R. Bachmann, T. H. Geballe and J. P. Maita, Phys. Rev. B, 2, 4580 (1970).

F. J. Morin and J. P. Maita, Phys. Rev. 129, 1115 (1963).

M. Murabayashi, Y. Takahashi and T. Mukaibo, J. Nucl. Sci. Technol. 7, 312 (1970),

P. F. Sullivan and G. Seidel, Phys. Rev. 173, 679 (1968).

in R. Fhrat and 1. Rinderer. Helv. Phys. Acta, 39. 566 (1966).

1 N. J. Trappeniers, Ber. Bunsenges. Phys. Chem. 70, 1080 (1970).

12 E. F. Westrum. Jr. et al. unpublished data.

13 T. F. Smith and N. E. Phillips. Chapter in Les Propriétés Physiques des Solides Sous Pression. Centre National de la Recherche Scientifique, Paris (1970), p. 191.

14 J. F. Martin. Chapter in Chemical Thermodynamics, Vol. I. The Chemical Society, London (1973), p. 133.

is C. Brot, J. Phys. (Paris) Colloque, 5a (supplément au no. 10, Tome 32), C5a-223 (1971).

16 C. Brot and M. Renaud, Bull. Soc. fr. Minéral. Cristallogr. 95, 183 (1972).

17 S. Hentiquez and E. F. Westrum. Jr., Presentation at the 1973 Calorimetry Conference, Worcester, Massachusetts.

18 S. C. Greer, J. M. H. Levelt Sengers and G. T. Furukawa, J. Chem. Phys. 57 (12), 5052 (1972).

19 M. J. Buckingham. Ber. Bunsenges. Phys. Chem. 76 (3-4), 287 (1972).

20 J. T. S. Andrews and W. E. Bacon, J. Chem. Thermodyn. 6, 515 (1974).

21 L. Misch and A. J. van der Wyk, Compt. Rend. Soc. Phys. Hist. Nat. Geneve, 54, 106 (1937).

22 H. H. Günthard, Pl. A. Plattner and E. Brandenberger, Experimentia, 4, 425 (1948).

${ }^{23} \mathrm{Y}$. Takeuchi and R. Pepinsky, Science, 124, $126(1956)$.

24 J. M. Robertson and H. M. Shearer, Nature, 177, 885 (1956).

25 J. D. Bernal, Nature, 178, 40 (1956).

26 J. M. Robertson, H. M. Shearer, G. A. Sim and D. G. Watson, Nature, 182, 177 (1958). 
27 J. M. Robertson, H. M. Shearer, G. A. Sim and D. G. Watson, Acta Cryst, 15, 1 (1962).

28 G. S. Pawley, Acta Cryst. 18, 560 (1965).

29 H. H. Günthard, Ph. D. Thesis, E. T. H. Zurich (1949),

30 E. Kovats, H. H. Günthard and Pl. A. Plattner, Helv. Chim. Acta, 38, 1912 (1955).

31 A. Bauder and H. H. Günthard, Helv. Chim. Acta, 45, 1698 (1962).

32 J. P. McCullough, H. L. Finke, J. F. Messerly, S. S. Todd, T. C. Kincheloe and G. Waddington, J. Phys. Chem. 61, 1105 (1957).

${ }^{33}$ C. A. Fyfe and G. J. Kupferschmidt, Can. J. Chem. submitted.

${ }^{34}$ A. van Tets and H. H. Günthard, Spectrochim. Acta, 19, 1495 (1963).

35 A. W. Hanson, Acta Cryst. 19, 19 (1965).

36 W. G. Lyon and E. F. Westrum, Jr., J. Chem. Thermodyn. 6, in press.

${ }^{37}$ E. F. Westrum, Jr. and F. Gronvold, J. Inorg. Nucl. Chem. 32, 2169 (1970).

38 J. R. Fletcher and F. W. Sheard, Solid State Commun. 9 (16), 1403 (1971).

${ }^{39}$ E. F. Westrum, Jr., Chapter on Thermodynamics of Crystals in Biennial Reviews of Science, Technology and Medicine, Physical Chemistry Series, Vol. X, ed. H. A. Skinner, Butterworths, London (1972).

40 J. P. Harrison, G. Lombardo and P. P. Peressini, J. Phys. Chem. Solids, 29, 557 (1968).

41 K. Andres, Phys, Rev. B 2, 3768 (1970).

42 G. Gilat, Phys. Rev. Letters, 23, 78 (1969).

43 B. H. Justice, E. F. Westrum, Jr., E. Chang and R. Radebaugh, J. Phys. Chem. 73, 333 (1969); cf. B. H. Justice and E. F. Westrum, Jr., J. Phys. Chem. 73, 1959 (1969) and earlier references.

44 J. R. Henderson, M. Muramoto and J. B. Gruber, J. Chem. Phys. 46, 2515 (1967).

45 P. Kisliuk, W. F. Krupke and J. B. Gruber, J. Chem. Phys. 40, 3606 (1964) ; J. B. Gruber, W. F. Krupke and J. M. Poindexter, J. Chem. Phys. 41, 3363 (1964).

46 D. Sh. Tsagareishvili and G. G. Gvelesiani. Teplofiz. Vys. Temp. 9.643 (1971).

47 W. N. Lawless, J. Phys. Chem. Solids, 30, 1161 (1969).

48 J. P. Harrison, P. P. Peressini and R. P. Pohl, Phys, Rev. 167, 856 (1968); G. R. Field and W. F. Sherman, J. Chem. Phys. 47, 2378 (1967); R. W. Dreyfus, J. Phys Chem. Solids, 29, 1941 (1968).

49 W. Reese, Phys. Rev. 181, 905 (1969).

50 B. Rolovs and T. Romanovskis, 1zv. Akad. Nauk SSSR. Ser, Fiz., 34, 2492 (1970).

s1 P. F. Milleron and G. Fournet, Elektrotech. Cas, 21, 257 (1970).

5.2 S. Grossmann and P. H. Richter, Phys. Letters, 33A. 39 (1970).

${ }_{54}$ G. J. Hyland, J. Solid State Chem. 2,318 (1970).

54 V. S. Neshpor and G. V. Samsonov, Izv. Vyssh. Ucheb. Zaved. Fiz. 12 , 23 (1969); Fiz. Metal. Metalloved 25, 1132 (1968).

55 E. F. Westrum. Jr. and F. Grønvold, J. Chem. Thermodyn. 1, 543 (1969).

$56 \mathrm{~J}$. J. Bartel and E. F. Westrum, Jr., Dopant Effects Upon the Verwey Transition in $\mathrm{Fe}_{3} \mathrm{O}_{4}$, from AIP Conference Proceedings, No. 10, Magnetism and Magnetic Materials (18th Annual Conference, Denver) (1973).

57 B. J. Evans and E. F. Westrum, Jr., Phys. Rev. B, 5 (9), 3791 (1972).

58 D. C. Herbert J. Phys. C, 3, 891 (1970).

59 H. E. Flotow, D. W, Osborne and E. F. Westrum, Jr., J. Chem. Phys. 49, 2438 (1968),

60 E. F. Westrum, Jr. and E. H. P. Cordfunke, unpublished data.

61 A. H. Cooke, Proceedings of the 11th International Conference on Low Temperature Physics, Vol. I, p 57 (1968) (St. Andrews, Scotland: Conference Organizing Committee).

12 N. P. Grazhdankina, Sov. Phys. Usp. 11, 727 (1969); Usp. Fiz. Nauk 96, 291 (1968).

63 T. A. Reichert and W. F. Giauque, J. Chem. Phys. 50, 4205 (1969).

${ }^{64}$ E. A. S. Lewis, Phys. Rev. B, 1, 4368 (1970).

65 D. L. Connelly, J. S. Loomis and D. E. Mapother, Phys. Rev. B, 3(3), 924 (1971).

66 W. G. Lyon and E. F. Westrum, Jr., J. Chem. Thermodyn. 6, in press.

${ }_{67}$ J. J. White, H. I. Song, J. E. Rives and D. P. Landau, Phys. Rev, B, 4(12), 4605 (1971).

68 J. J. White, III, Paper presented at the Confereno on Phase Transitions and Their Applications in Materials Science, University Park, Pa., May 1973.

69 R. A. Bari and J. Sivardière, Phys. Rev. B, 5(11), 4466 (1972).

${ }^{70}$ T. Schneider, G. Srinivasan and C. P. Enz, Phys, Rev. A, 5(3), 1528 (1972).

71 L. Brewer, Science, 161, 115 (1968).

${ }^{72} \mathrm{~L}$. Brewer, Thermodynamics and Alloy Behavior of the BCC and FCC Phases of Plutonium and Thorium, published in Plutonium 1970 and Other Actinides. TMS Nuclear Metallurgy Series, 


\section{CALORIMETRY OF PHASE AND ORDERING TRANSITIONS}

Vol. XVII, Part II. W. N. Miner, ed., Metallurgical Society of America. Institute of Mining, Metallurgical and Petroleum Engineers (1970).

73 L. Brewer, J. Opt. Soc. Am. 61, 1101 (1971).

${ }^{74}$ L. Brewer and P. R. Wengert, Metall. Trans. 4, 83 (1973).

75 A. R. Ubbelohde, Melting and Crystal Structure, Clarendon Press, Oxford (1965).

76 R. Blachnik and A. Schneider, Z. Anorg. Allg. Chem. 372, 314 (1970).

77 Phase Transformations. Proceedings of a Seminar of the American Society for Metals, October 1968, American Society for Metals: Metals Park, Ohio (1970).

${ }^{78}$ R. S. Carter and J. J. Rush, Molecular Dynamics and Structure of Solids, U.S. Government Printing Office, Washington (1969).

79 Phase Transitions. Proceedings of the Fourteenth Conference on Chemistry at the University of Brussels, May 1969, Wiley, London (1971).

80 Proceedings of the First International Conference on Calorimetry and Thermodynamics at Warsaw, 1969, Polish Scientific Publishers, Warsaw (1970).

81 Structural Phase Transitions and Soft Modes. Conference Proc. NATO Advanced Study Institute, Geilo, Norway, 13-20 April, 1971. E. J. Samuelson, E. Anderson and J. Feder, eds., Universitetsforlaget, Oslo (1971).

82 Phase Transitions. Proceedings of the Fourteenth Conference on Chemistry at the University of Brussels, May 1969, Interscience, London (1971).

83 S. A. Rice, Phase Transitions, Proceedings of the 14th Conference on Chemistry at the University of Brussels, May 1969, Interscience, London (1971).

${ }^{5} \downarrow$ C. W. F. T. Pistorius, J. Chem. Phys. 51(6), 2604 (1969).

85 C. Landee and E. F. Westrum, Jr., unpublished results.

86 R. Carling and E. F. Westrum, Jr., unpublished results.

${ }^{87}$ B. N. Sudaridov et al. Zhur. Fiz. Khim. 74, 37 (1970).

ss J. J. Bartel, A. Alles and E. F. Westrum. Jr., unpublished data.

89 N. J. Trappeniers, Ber. Bunsenges Phys. Chem. 70, 1080 (1966).

90 E. B. Amitin, Yu. A. Kovalevskaya and I. E. Paukov, Soviet Phys. Solid State, 14, 2902 (1973).

91 R. H. Brout, Phase Transitions, W. A. Benjamin, New York (1965).

92 H. E. Stanley, Introduction to Phase Transitions and Critical Phenomena. Oxford University Press. New York (1971).

93 Phase Transitions and Critical Phenomena, Vol. I, eds. C. Domb and M. S. Green, Academic Press, New York (1972).

94 Phase Transitions and Critical Phenomena, Vol. II, eds. C. Domb and M. S. Green, Academic Press. New York (1972).

95 Cooperative Phenomena Nea Phase Transitions, A Bibliography With Selected Readings, ed. H. E. Stanley, MIT Press, Cambridge, Mass. (1973).

96 A. Jayaraman. Ann. Rev. Mater. Sci. 2121 (1972). 